\title{
Multiscaled Cross-Correlation Dynamics on SenseCam Lifelogged Images
}

\author{
N. $\mathrm{Li}^{1,2}$, M. Crane ${ }^{1}$, H.J. Ruskin ${ }^{1}$, C. Gurrin ${ }^{2}$ \\ 1 Centre for Scientific Computing \& Complex Systems Modelling \\ ${ }^{2}$ CLARITY: Centre for Sensor Web Technologies \\ School of Computing, Dublin City University, Ireland \\ na.li@dcu.ie, \{mcrane, hruskin, cgurrin\}@computing.dcu.ie
}

\begin{abstract}
In this paper, we introduce and evaluate a novel approach, namely the use of the cross correlation matrix and Maximum Overlap Discrete Wavelet Transform (MODWT) to analyse SenseCam lifelog data streams. SenseCam is a device that can automatically record images and other data from the wearer's whole day. It is a significant challenge to deconstruct a sizeable collection of images into meaningful events for users. The cross-correlation matrix was used, to characterise dynamical changes in non-stationary multivariate SenseCam images. MODWT was then applied to equal-time Correlation Matrices over different time scales and used to explore the granularity of the largest Eigenvalue and changes, in the ratio of the sub-dominant Eigenvalue spectrum dynamics, over sliding time windows. By examination of the eigenspectrum, we show that these approaches can identify "Distinct Significant Events" for the wearers. The dynamics of the Eigenvalue spectrum across multiple scales provide useful insight on details of major events in SenseCam logged images.
\end{abstract}

Keywords: Lifelogging; SenseCam; Images; equal-time Correlation Matrices; Maximum Overlap Discrete Wavelet Transform

\section{Introduction}

In Lifelogging, the subject typically wears a device to record episodes of their daily lives. This concept has been pioneered to the extent of including a wearable computer, camera and viewfinder with wireless internet connection in order to capture personal activities through the medium of images or video. Developed by Microsoft Research in Cambridge, UK, SenseCam [1] is such a camera worn around the neck to capture images and other sensor readings automatically, in order to record the wearer's every moment. Such images and other data can be periodically reviewed to refresh and strengthen the wearer's memory. The device takes pictures at VGA resolution, (480x640 pixels), and stores these as compressed JPEG files on internal flash memory. SenseCam can collect a large amount of data, even over a short period of time, with a picture typically taken every 30 seconds. Hence there are about 4,000 images captured in any one day, or of the order of 1 million images captured per year. 
Although research shows that the SenseCam can be an effective memory-aid device [2, 3], as it helps to improve retention of an experience, wearers seldom wish to review life events by browsing large collections of images manually [4-7]. The challenge then is to manage, organise and analyse these large image collections in order to automatically highlight key episodes and, ideally, classify these in order of importance to the wearer. Previously, the lifelog of SenseCam images has been segmented into approximately 20 distinct events in a wearer's day, or about 7,000 events per year [8], but this large collection of personal information still contains a significant percentage of routine events. The challenge is to determine which events are the most important or unusual to the wearers.

In recent years, the behaviour of the largest Eigenvalue of a cross-correlation matrix over small windows of time, has been studied extensively, e.g. for financial series [9-20], electroencephalographic (EEG) recordings [21, 22], magnetoencephalographic (MEG) recordings [23] and a variety of other multivariate data. In this paper, we investigate the same approach to analysis SenseCam lifelog data streams. We aim to apply the multiscaled cross-correlation matrix technique to study the dynamics of the SenseCam images, where this time series should exhibit atypical or non-stationary, characteristics, which highlight "Distinct Significant Events" in the data. We also evaluate our approach by identifying the boundaries between different daily events, which might include working at the office, walking outside, shopping etc. We found that different distinct events or activities can be detected at different scales.

This paper is organized as follow: in Section 2, we review the methods, in Section 3 we describe the data used, while Section 4 details with results obtained. Conclusions are given in Section 5.

\section{Methods}

Our previous research [24] has shown that SenseCam image time series reflect strong long-range correlation, indicating that the time series is not a random walk, but is cyclical, with continuous low levels of background information picked up constantly by the device. In this section, we first use equal-time cross-Correlation Matrices to characterise dynamical changes in non-stationary multivariate SenseCam time-series. The Maximum Overlap Discrete Wavelet Transform (MODWT) is then used to calculate equal-time Correlation Matrices over different time scales. This enables exploration of details of the Eigenvalue spectrum and in particular, examination of whether specific events show evidence of distinct signatures at different time scales.

\subsection{Correlation Dynamics}

The equal-time cross-correlation matrix can used to characterise dynamical changes in non-stationary multivariate time series. Before examining the image time series in detail, it is important to introduce the gray scale pixel values concept. In a gray scale 
image, a pixel with a value of 0 is completely black and a pixel with a value of 255 is completely white. While images captured from SenseCam are coloured, these are converted to gray-scale images in order to simplify the calculation. To reduce the size of the calculation further and the amount of memory used, we first adopt an averaging method to decrease image size from $480 \times 640$ pixels to $6 \times 8$ pixels. Hence the correlation matrix is made up of 48 time series for over 10,260 images. Given pixels $G_{i}(t)$, of a collection of images, we normalize $G_{i}$ within each window in order to standardize the different pixels for the images as follows:

$$
g_{i}(t)=\frac{G_{i}(t)-\overline{G_{i}(t)}}{\sigma_{(i)}}
$$

where $\sigma_{(i)}$ is the standard deviation of $G_{i}$ for image numbers $i=1, \ldots, N$, and $\overline{G_{i}}$ is the time average of $G_{i}$ over a time window of size $T$. Then the equal-time cross-correlation matrix may be expressed in terms of $g_{i}(t)$

$$
C_{i j} \equiv\left\langle g_{i}(t) g_{j}(t)\right\rangle
$$

The Eigenvalues $\lambda_{i}$ and eigenvectors $\bar{v}_{i}$ of the correlation matrix $C$ are found from the Eigenvalue equation $C \overline{v_{i}}=\lambda_{i} \overline{v_{i}}$.

\subsection{Wavelet Multiscale analysis}

The Maximum Overlap Discrete Wavelet Transform (MODWT) [25-28], is a linear filter that transforms a series into coefficients related to variations over a set of scales. It produces a set of time-dependent wavelet and scaling coefficients with basis vectors associated with a location $t$ and a unitless scale $\tau_{j}=2^{j-1}$ for each decomposition level $j=1, \ldots, J_{0}$. Unlike the DWT, the MODWT, has a high level of redundancy. However, it is non-orthogonal and can handle any sample size $N$, whereas the DWT restricts the sample size to a multiple of $2^{j}$. MODWT retains downsampled ${ }^{3}$ values at each level of the decomposition that would be discarded by the DWT. This reduces the tendency for larger errors at lower frequencies when calculating frequency dependent variance and correlations, as more data are available. For MODWT the $j^{\text {th }}$ level equivalent filter coefficients have a width $L_{j}=\left(2^{j}-1\right)(L-1)+1$, where $L$ is the width of the $j=1$ base filter.

Decomposing a signal using the MODWT to $J$ levels theoretically involves the application of $J$ pairs of filters. The filtering operation at the $j^{\text {th }}$ level consists of applying a rescaled father wavelet to yield a set of detail coefficients

$$
\tilde{D}_{j, t}=\sum_{l=0}^{L_{j}-1} \tilde{\varphi}_{j, l} f_{t-l}
$$

\footnotetext{
${ }^{3}$ Downsampling or decimation of the wavelet coefficients retains half of the number of coefficients that were retained at the previous scale. Downsampling is applied in the Discrete Wavelet Transform
} 
and a rescaled mother wavelet to yield a set of scaling coefficients

$$
\tilde{S}_{j, t}=\sum_{l=0}^{L_{j}-1} \tilde{\phi}_{j, l} f_{t-l}
$$

for all times $t=\ldots,-1,0,1, \ldots$, where $f$ is the function to be decomposed [29]. The rescaled mother, $\tilde{\varphi}_{j, t}=\frac{\varphi_{j, t}}{2^{j}}$, and father, $\tilde{\phi}_{j, t}=\frac{\varphi_{j, t}}{2^{j}}$, wavelets for the $j^{t h}$ level are a set of scale-dependent localized differencing and averaging operators and can be regarded as rescaled versions of the originals. The $j^{t h}$ level equivalent filter coefficients have a width $L_{j}=\left(2^{j}-1\right)(L-1)+1$, where $L$ is the width of the $j=1$ base filter. In practice the filters for $j>1$ are not explicitly constructed because the detail and scaling coefficients can be calculated, using an algorithm that involves the $j=1$ filters operating recurrently on the $j^{\text {th }}$ level scaling coefficients, to generate the $j+1$ level scaling and detail coefficients [29].

The wavelet variance $\nu_{f}^{2}\left(\tau_{j}\right)$ is defined as the expected value of $\tilde{D}_{j, t}^{2}$ if we consider only the non-boundary coefficients. An unbiased estimator of the wavelet variance is formed by removing all coefficients that are affected by boundary conditions and is given by

$$
\nu_{f}^{2}\left(\tau_{j}\right)=\frac{1}{M_{j}} \sum_{t=L_{j}-1}^{N-1} \tilde{D}_{j, l}^{2}
$$

where $\tilde{D}_{j, l}$ is a rescaled father wavelet, which yields a set of scaling coefficients, $M_{j}=$ $N-L_{j}+1$ is the number of non-boundary coefficients at the $j^{t h}$ level.

The wavelet covariance between functions $f(t)$ and $g(t)$ is similarly defined to be the covariance of the wavelet coefficients at a given scale. The unbiased estimator of the wavelet covariance at the $j^{\text {th }}$ scale is given by

$$
\nu_{f g}\left(\tau_{j}\right)=\frac{1}{M_{j}} \sum_{t=L_{j}-1}^{N-1} \tilde{D}_{j, l}^{f(t)} \tilde{D}_{j, l}^{g(t)}
$$

The MODWT estimate of the wavelet cross-correlation between functions $f(t)$ and $g(t)$ may be calculated using the wavelet covariance and the square root of the wavelet variance of the functions at each scale $j$. The MODWT estimator, of the wavelet correlation is thus given by

$$
\rho_{f g}\left(\tau_{j}\right)=\frac{\nu_{f g}\left(\tau_{j}\right)}{\nu_{f}\left(\tau_{j}\right) \nu_{g}\left(\tau_{j}\right)}
$$

where $\nu_{f g}\left(\tau_{j}\right)$ is the covariance between $f(t)$ and $g(t)$ at scale $j, \nu_{f}\left(\tau_{j}\right)$ is the variance of $f(t)$ at scale $j$ and $\nu_{g}\left(\tau_{j}\right)$ is the variance of $g(t)$ at scale $j$.

The multiscaled cross-correlation matrix technique is adopted in order to help highlight non-stationary events (in SenseCam lifelog data streams), which could be of importance. 


\section{Data}

In this study, the data were generated from one person wearing the SenseCam over a six day period, from a Saturday to a Thursday. These particular days were chosen in order to include a weekend, where normal home activity varies in comparison to events on weekdays or a working week. Forming a total lifelog of 10,260 images, with average wearing time varying from about 11 hours on Saturday to about 6 hours on Tuesday. Saturday involved the subject walking to the nearest bus stop from home, a bus journey to the city centre, walking through local streets as well as a visit to a shopping centre. This day also involved dinner with a friend and a bus journey back to the original bus stop. Over the next five days, these images described a typical day for the subject: sitting in the office, talking with a colleague and sharing lunch in the cafeteria, the journey from the office to home, and the next morning from home to their office and so on. Figure 1 shows some examples of SenseCam images. Data statistics are reported in Table 1 . To create a ground truth, the user reviewed her collection and manually marked the boundary image between all events.
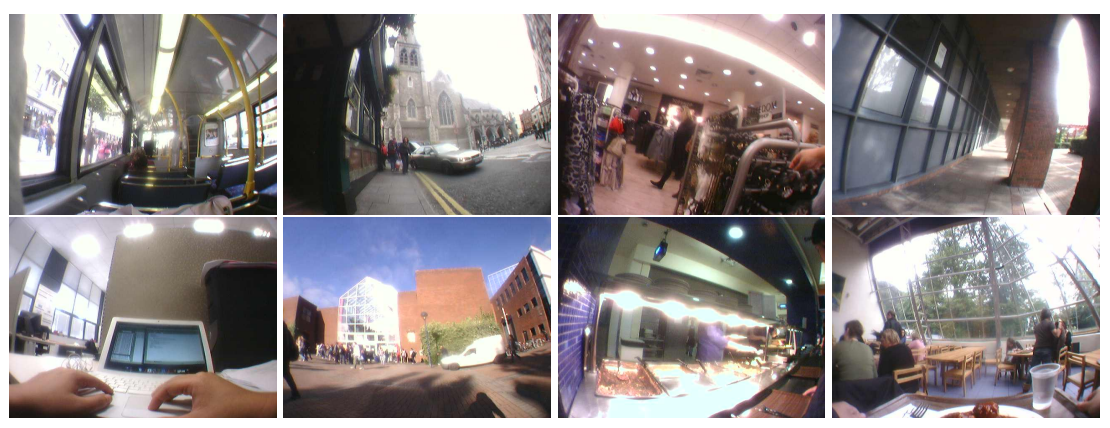

Fig. 1. Example of SenseCam Images

Table 1. Data Statistics

\begin{tabular}{lccc}
\hline User Events Catalogue & Groundtruthed Events & Images \\
\hline 1 & Working & 15 & 6146 \\
1 & Walking Outside & 32 & 1494 \\
1 & Shopping & 12 & 826 \\
1 & Eating & 3 & 658 \\
1 & Taking Bus & 2 & 297 \\
1 & Others & 5 & 839 \\
\hline & Total: 69 & Total: 10,260 \\
\hline
\end{tabular}




\section{Results}

\subsection{Dynamics of the largest Eigenvalue for different window sizes}

In financial data, it has been known for some time that the largest Eigenvalue $\left(\lambda_{1}\right)$ contains information on risk associated with the particular assets of which the covariance matrix is comprised, (i.e. the 'market' factor) [30]. Similarly we would expect the largest Eigenvalue to present information from the image that reflects the largest change in the SenseCam recording.
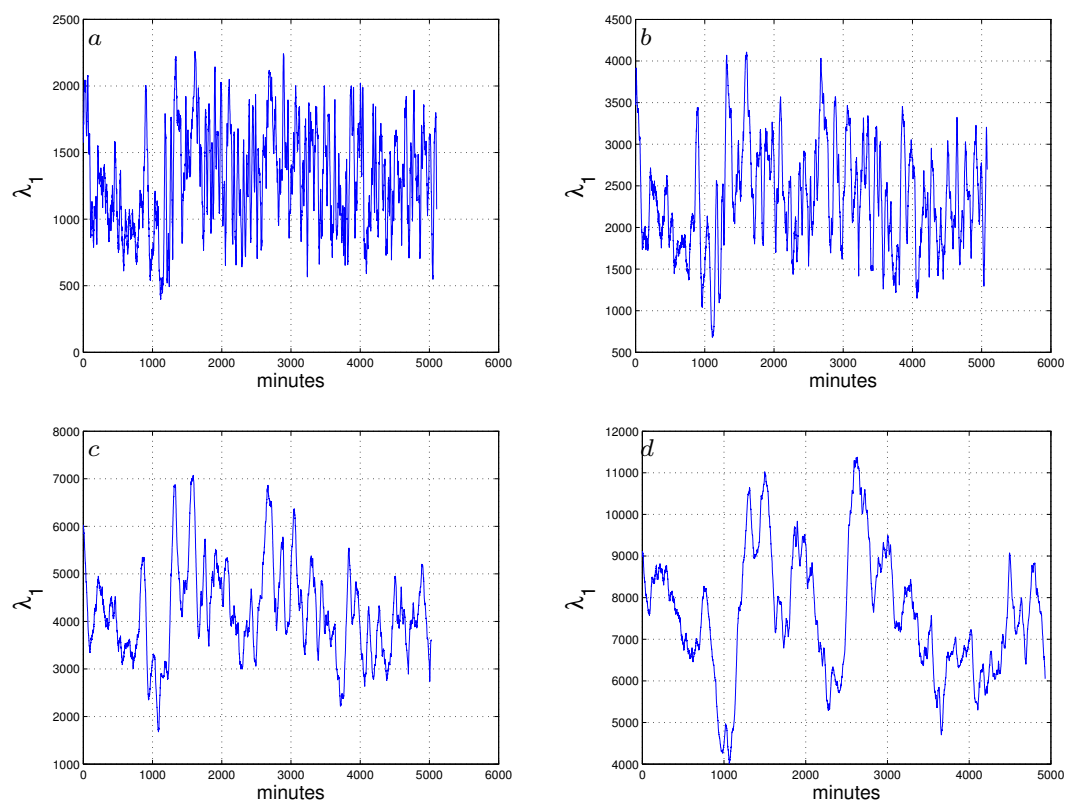

Fig. 2. Total 10260 Images (size: 6x8 pixels) largest Eigenvalue Distribution using a sliding window of $50 \operatorname{Images}(a), 100 \operatorname{Images}(b), 200 \operatorname{Images}(c)$ and $400 \operatorname{Images}(d)$

Typically SenseCam captures two images every minute, so we can measure wavelet Eigenvalue dynamics in time (minutes). Figure 2 shows the time series of the largest Eigenvalue for different window sizes. From these, we note the following features:

- With increased window size comes increased smoothing - as expected. This removes some of the high frequency small-scale changes, typically associated with noise.

- As the window size is increased, the peaks in the series become more pronounced. These peaks reflect large changes in greyscale of the images. 


\subsection{Wavelet analysis}

For the present study, we selected the least asymmetric (LA) wavelet, (known as the Symmlet, [31]), which exhibits near symmetry about the filter midpoint. LA filters are defined in even widths and the optimal filter width is dependent on the characteristics of the signal and the length of the data series. The filter width chosen for this study was the LA8, (where 8 refers to the width of the scaling function), since this enables accurate calculation of wavelet correlations to the $10^{\text {th }}$ scale, which is appropriate given the length of data series available. Although the MODWT can accommodate any level, $J_{0}$, the largest level, is chosen in practice, so as to prevent decomposition at scales longer than the total length of the data series, (hence the choice of the $10^{\text {th }}$ ), while still containing enough detail to capture subtle changes in the signal, [29].

First, the MODWT of the pixels for each image was calculated within each window and the correlation matrix between pixels at each scale found. The Eigenvalues of the correlation matrix in each window were determined, and the Eigenvalue time series were normalised in time. Then the largest Eigenvalue for different window sizes was analysisd. These results are shown in a heat map in Figure 3 and discussed below.

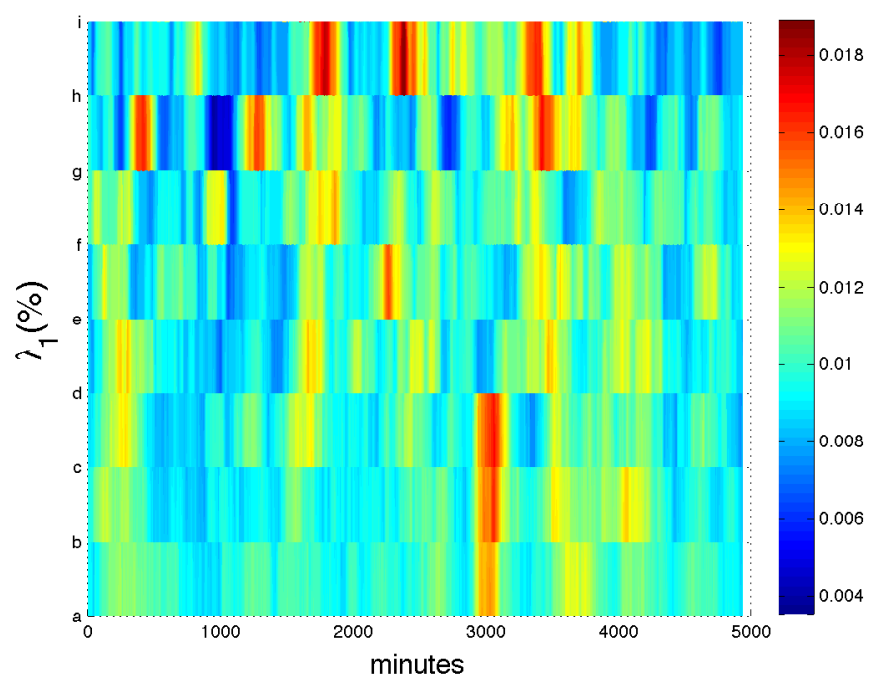

Fig. 3. Heatmap diagram showing the dynamics of the largest Eigenvalue $\lambda_{1}$ across 9 wavelet scales. Scales $1(a)$ to $9(i)$ correspond to a 1-2 minute period, a 2-4 minute period, a 4-8 minute period, a 8-16 minute period, a 16-32 minute period, a 32-64 minute period, a 64-128 minute period, a 128-256 minute period, a 256-512 minute period. 
Dynamics of the largest Eigenvalue at various wavelet scales Figure 3 shows the time series of the largest Eigenvalue dynamics across different wavelet scales. Some peaks are consistently captured by the SenseCam at certain scales, such as a peak around 3000 minutes, (captured by wavelet scales 1,2, 3 and corresponding to a 1-2 minute period, a 2-4 minute period and a 4-8 minute period). These peaks should help us to identify major events or activities in the data. The different features, found at various scales, suggest that the correlation matrix captured different major events with different time horizons. This will be examined in more detail in the next subsection.

The largest Eigenvalue $\lambda_{1}$ compared with the ratio of $\lambda_{1} / \lambda_{2}$ dynamics We also wish to ascertain whether the sub-dominant Eigenvalues $\lambda_{2}$ hold further information on the key sources or major events and what information these contribute additionally to the images. The dynamics of the largest Eigenvalue and changes in the ratio of the largest Eigenvalues were examined from a MODWT analysis. Here, we detail several scenarios for the peaks in the largest Eigenvalue and the ratio Eigenvalues for a window size of 400 images. We have tried to identify the position and nature of peak sources or major events from the real images generated from SenseCam collections.

In Figure 4, the dynamics of the series for the largest Eigenvalue and changes of the Eigenvalue ratio $\lambda_{1} / \lambda_{2}$ were examined for the MODWT analysis. The technique gives a clear picture of the movements in the image time series by reconstructing them using each wavelet component. MODWT captured the particular marked features at specified scales. A number of features from the image are reproduced and can be examined by studying these Eigenvalue series.

We have detailed the scenario for each peak in Figure 4. The subject was sitting in front of her laptop, with laptop, lights and seating position unchanged over on extended period, contributing high pixel values in a sequence of images. This typical case was always marked by a peak in the SenseCam signal. The signal fluctuation is caused by changes, such as the subject moving from indoors to outdoors, light level alternatives, the subject changing position from sitting to moving, movement increase and more people joining in the scene. Note that movement increase or multiple person interactions can be captured by specific scales, using the MODWT method. The ratio analysis strongly reinforces observations on the largest Eigenvalue over time. The ratio of $\lambda_{1} / \lambda_{2}$ has smaller variation compared to that for the largest Eigenvalue $\lambda_{1}$. This implies that the second largest Eigenvalue $\left(\lambda_{2}\right)$ carries additional information on the description of, but does not contribute in large part to occurrence of the major events for SenseCam. It does carry information for events surrounding the major ones, e.g. possible lead-in, lead-out. 

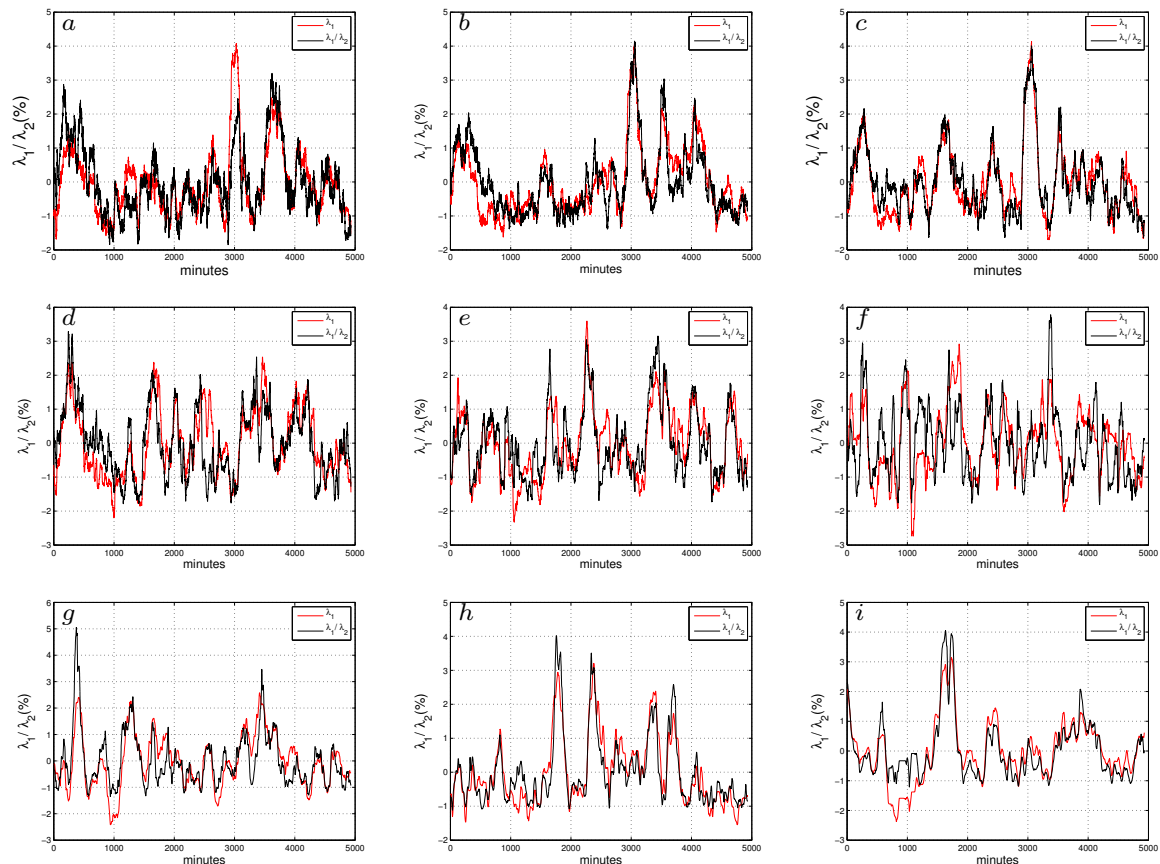

Fig. 4. The largest Eigenvalue $\lambda_{1}$ (red) and the ratio of $\lambda_{1} / \lambda_{2}$ (black) dynamics across 9 wavelet scales. The Top 3 figures $(a-c)$ are for scales 1 to 3 corresponding to a 1-2 minute period, a 2-4 minute period, a 4-8 minute period, Middle 3 figures ( $d-f)$ are for scales 4 to 6 corresponding to a 8-16 minute period, a 16-32 minute period, a 32-64 minute period and Bottom 3 figures $(g-i)$ are for scales 7 to 9 corresponding to a 64-128 minute period, a 128-256 minute period, a 256-512 minute period.

\subsection{Evaluation}

We evaluate the different wavelet scales performance using the precision, $P$, and recall, $R$ metrics, as defined below. Moreover, we compute the $F_{1}$ score is a measure of a method's accuracy [32].

$$
\begin{gathered}
P=\frac{\mid \text { determined boundaries }|-| \text { wrong boundaries } \mid}{\mid \text { determined boundaries } \mid} \\
R=\frac{\mid \text { detected reference boundaries } \mid}{\mid \text { determined boundaries } \mid} \\
F_{1}=2 * \frac{P * R}{P+R}
\end{gathered}
$$

Table 1 shows more than 60 groundtruthed events manually segmented by a user. In order to determine accurate boundary, each peak point boundary is calculated, (for the 
difference between neighboring left and right most trough values) [8]. This is a crude boundary designation, all values within a peak area are combined so that a signal value is less informative. Significant peaks are determined (distinct events or activities) by $\lambda_{1} / \lambda_{2}$ percentage pixel values are larger than zero. Table 2 shows the precision, recall and $F_{1}$ measure for $\lambda_{1} / \lambda_{2}$ at different wavelet scales. As we can see, all scales appear with high precision but very low recall. The main weakness as well as strength for wavelet scales is that different scales highlight different distinct events dependent on the time horizons. Some events at certain scales will be missed, so that the overall recall values are low for this approach. In addition, some activities such as working in front of the laptop last for several hours. In manually segmenting 69 events of 10,260 images only, the detection probability for a given event is quite low. In consequence this approach is quite crude and suggest that further modification needed, such as incorporating other than peak distance an weighting scale combinations.

Table 2. Precision, Recall and $F_{1}$ measures for MODWT method

\begin{tabular}{lccc}
\hline & \multicolumn{3}{c}{$\lambda_{1} / \lambda_{2}$} \\
\hline Wavelet Scales & $P$ & $R$ & $F_{1}$ \\
\hline Scale1 (1-2 minute period) & 0.3929 & 0.4058 & 0.3992 \\
Scale2 (2-4 minute period) & 0.7857 & 0.2029 & 0.3225 \\
Scale3 (4-8 minute period) & 0.5000 & 0.3188 & 0.3894 \\
Scale4 (8-16 minute period) & 0.4783 & 0.3333 & 0.3929 \\
Scale5 (16-32 minute period) & 0.5238 & 0.3043 & 0.3850 \\
Scale6 (32-64 minute period) & 0.5789 & 0.2754 & 0.3732 \\
Scale7 (64-128 minute period) & 0.7333 & 0.2174 & 0.3354 \\
Scale8 (128-256 minute period) & 0.9167 & 0.1739 & 0.2924 \\
Scale9 (256-512 minute period) & 1 & 0.1594 & 0.2750
\end{tabular}

\section{Conclusions}

The Maximum Overlap Discrete Wavelet Transform (MODWT) method, calculating equal-time Correlation Matrices over different time scales, was used to investigate the largest Eigenvalue and the changes in the sub-dominant Eigenvalue ratio spectrums. As shown in Figure 3, the different features, found at various scales, suggest that the correlation matrix captured different major events with different time horizons. We note that these "jitters" may contain additional information surrounding the major events. This suggests that the correlation matrix for different information captured from SenseCam can be filtered by different time horizons. Those consistently occurring peaks should help us to identify major events captured by the SenseCam. By examining the behaviour of the largest Eigenvalue and the change in Eigenvalue ratios over time, the Eigenvalue ratio analysis confirmed that the largest Eigenvalue carries most of the major event information, whereas subsequent Eigenvalues carry information on supporting or lead in/ lead out events. On analyzing major events, (with a sliding window set to 400 images), 
we identified the light level as a major event delineator during static periods of image sequence. While the methods set out in this paper appear to perform quite well for precision value, they do quite poorly for the recall value at different time scales. The value in the method lies in the fact that prior information about events can be used as an additional filter. An example of this is that sitting in front of a laptop is likely to show up in the higher scaled wavelet scales. Overall the MODWT method provides a powerful tool for examination of the nature of the captured SenseCam data for certain categories of users.

Future work includes checking larger datasets and multiple users to help to confirm initial findings which are clearly capable of refinement, as mentioned Section 4. Studying the multi-scaled correlation dynamics over multiple sensors may be useful to detect distinct events or activities and could also be worthwhile for classification of event type in SenseCam data.

\section{Acknowledgments}

NL would like to acknowledge generous support from the Sci-Sym Centre Small Scale Research Fund, as well as additional support from the School of Computing and CLARITY Centre.

\section{References}

1. S. Hodges, L. Williams, E. Berry, S. Izadi, J. Srinivasan, A. Butler, G. Smyth, N. Kapur and K. Wood: SenseCam: A Retrospective Memory Aid, In UbiComp: 8th International Conference on Ubiquitous Computing, volume 4602 of LNCS, California, USA, (2006) 177 193.

2. R. Harper, D. Randall, N. Smyth, C. Evans, L. Heledd and R. Moore.: Thanks for the Memory, In HCI 2007-Proceedings of the 21st BCS HCI Group Conference, Lancaster, U.K., (2007)

3. R. Harper, D. Randall, N. Smyth, C. Evans, L. Heledd and R. Moore.: The Past is a Different Place: They Do Things Differently There, In Designing Interactive Systems, Cape Town, South Africa, (2008) 271-280

4. D. Ashbrook, K. Lyons and J. Clawson.: Capturing Experiences Anytime, Anywhere, IEEE Pervasive Computing, (2006)

5. G. Bell and J. Gemmell: A Digital Life, Scientific American, (2007)

6. M.L. Lee and A.K. Dey: Providing good memory cues for people with episodic memory impairment, In Assets'07: Proceedings of the 9th international ACM SIGACCESS conference on Computers and accessibility, Tempe, Arizona, USA, (2007) 131-138

7. W.H. Lin and A. Hauptmann: Structuring Continuous Video Recordings of Everyday Life Using Time-Constrained Clustering, In Multimedia Content Analysis, Management and Retieval SPIE-IST Electronic Imaging, volume 6073, San Jose, California, USA, (2006) 111-119

8. A.R. Doherty and A. F. Smeaton: Automatically Segmenting Lifelog Data into Events, Proc. WIAMIS' 08, (2008) 20-23 
9. L. Laloux, P. Cizeau, J.-P Bouchaud and M. Potters: Noise dressing of financial correlation matrices, Phys. Rev. Lett. 83(7) (1999) 1467-1470

10. V. Plerou, P. Gopikrishnan, B. Rosenow, L.A. Nunes Amaral and H.E. Stanley.: Universal and non-uiversal properties of cross-correlations in financial time series, Phys. Rev. Lett. 83(7) (1999) 1471-1474

11. L. Laloux, P. Cizeau, J.-P. Bouchaud and M. Potters: Random matrix theory and financial correlations, Int. J. Theoret. Appl. Finance 3 (3) (2000) 391-397.

12. V. Plerou, P. Gopikrishnan, B. Rosenow, L.A. Nunes Amaral, T. Guhr and H.E. Stanley.: A random matrix approach to cross-correlations in financial data, Phys. Rev. E 65 (2000) 066126/1-066126/18

13. P. Gopikrishnan, B. Rosenow, V. Plerou and H. Eugene Stanley.: Indentifying business sectors from stock price fluctuations, Phys. Rev. E 64 (2001) 035106R/1-035106R/4

14. A. Utsugi, K. Ino and M. Oshikawa: Random matrix theory analysis of cross-correlations in financial markets, Phys. Rev. E 70 (2004) 026110/1-026110/17

15. J.P. Bouchaud and M. Oshikawa: Theory of Financial Risk and Derivative Pricing, Cambridge University Press, (2003)

16. D. Wilcox and T. Gebbie: On the analysis of cross-correlations in South African market data, Physica A 344 (1-2) (2004) 294-298

17. S. Sharifi, M. Crane, A. Shamie and H.J. Ruskin: Random matrix theory for portfolio optimization: A stability approach, Physica A 335 (3-4) (2004) 629-643

18. T. Conlon, H.J. Ruskin and M. Crane.: Random matrix theory and fund of funds portfolio optimisation, Physica A 382 (2) (2007) 565-576

19. T. Conlon, H.J. Ruskin and M. Crane: Wavelet multiscale analysis for Hedge Funds: Scaling and Strategies, Physica A (2008) 5197-5204

20. B. Podobnik and H.E. Stanley: Detrended cross-correlation analysis: A new method for analysing two non-stationary time series, Phys. Rev. Lett. (2008) 100(8):084102/1$084102 / 11$

21. K. Schindler, H. Leung, C. E. Elger and K. Lehnertz.: Assessing seizure dynamics by analysing the correlation structure of multichannel intracranial EEG, Brain 130 (2007) 65-77

22. K. Schindler, C.E. Elger and K. Lehnertz: Increasing synchronization may promote seizure termination: Evidence from status epilepticus, Clin, Neurophysiol. 118 (9) (2007) 1955-1968

23. J. Kwapien, S. Drozda and A.A. Ionannides: Temporal correlations versus noise in the correlation matrix formalism: An example of the brain auditory response, Phys. Rev. E 62 (2000) 5557-5564

24. N. Li, M. Crane and Heather J. Ruskin: Automatically detecting "significant events" on sensecam. ERCIM News (87) (2011)

25. C.-K. Peng, S.V. Buldyrev, S. Havlin, M. Simons, H.E. Stanley and A.L. Golderberger.: On the mosaic organization of DNA sequences, Phys. Rev. E 49 (1994) 1685-1689

26. J.A.O. Matos, S.M.A. Gama, H.J. Ruskin, A.A. Sharkasi and M. Crane: An econophysics approach to the Portuguese Stock Index-PSI-20, Physica A 342 (2004) 665-676

27. S.V. Buldyrev, A.L. Goldberger, S. Havlin, R.N. Mantegna, M.E. Matsa, C.-K. Peng, M. Simons and H.E. Stanley: Long-range correlation properties of coding and noncoding DNA sequences: GenBank analysis, Phys. Rev. E 51 (1995) 5084-5091

28. C. Heneghan and G. McDarby: Establishing the relation between detrended fluctuation analysis and power spectral density analysis for stochastic processes, Phys. Rev. E 62 (2000) 6103-6110

29. D.B. Percival and A.T. Walden: Wavelet methods for time series analysis, Cambridge University press, (2000)

30. A. Sharkasi, M. Crane, H.J. Ruskin and J.A. Matos: The reaction of stock markets to crashes and events: A comparison study between emerging and mature markets using wavelet transforms, Physica A 368, (2006) 511-521 
31. C.S. Burrus, R.A. Gopinath and H. Gao: Introduction to wavelets and wavelets transforms, Prentice Hall, (1997)

32. H. Misra, F. Hopfgartner, A. Goyal, P. Punitha and J. M. Jose: TV news story segmentation based on semantic coherence and content similarity. MMM (2010) 347-357 